14. Zimmerman, L. B., De Jesus-Escobar, J. M. \& Harland, R. M. The Spemann organizer signal noggin binds and inactivates bone morphogenetic protein 4. Cell 86, 599-606 (1996).

15. Hsu, D. R., Economides, A. N., Wang, X., Eimon, P. M. \& Harland, R. M. The Xenopus dorsalizing factor gremlin identifies a novel family of secreted proteins that antagonize BMP activities. Mol. Cell 1, 673-683 (1998).

16. Capdevila, J., Tsukui, T., Rodriquez Esteban, C., Zappavigna, V. \& Izpisua Belmonte, J. C. Control of vertebrate limb outgrowth by the proximal factor Meis2 and distal antagonism of BMPs by gremlin. Mol. Cell 4, 839-849 (1999).

17. Minabe-Saegusa, C., Saegusa, H., Tsukahara, M. \& Noguchi, S. Sequence and expression of a nove mouse gene PRDC (protein related to DAN and cerberus) identified by a gene trap approach. Dev. Growth Differ. 40, 343-353 (1998)

18. Brunkow, M. E. et al. Bone dysplasia sclerosteosis results from loss of the SOST gene product, a nove cystine knot-containing protein. Am. J. Hum. Genet. 68, 577-589 (2001).

19. Gazzerro, E., Gangji, V. \& Canalis, E. Bone morphogenetic proteins induce the expression of noggin which limits their activity in cultured rat osteoblasts. J. Clin. Invest. 102, 2106-2114 (1998).

20. Greenwald, J. A. et al. In vivo modulation of FGF biological activity alters cranial suture fate. Am J. Pathol. 158, 441-452 (2001).

21. Moore, R., Ferretti, P., Copp, A. \& Thorogood, P. Blocking endogenous FGF-2 activity prevents cranial osteogenesis. Dev. Biol. 243, 99-114 (2002).

22. Mansukhani, A., Bellosta, P., Sahni, M. \& Basilico, C. Signaling by fibroblast growth factors (FGF) and fibroblast growth factor receptor 2 (FGFR2)-activating mutations blocks mineralization and induces apoptosis in osteoblasts. J. Cell Biol. 149, 1297-1308 (2000).

23. Bradley, J. P. et al. Studies in cranial suture biology: in vitro cranial suture fusion. Cleft PalateCraniofac. J. 33, 150-156 (1996).

24. Dixon, M. E., Armstrong, P., Stevens, D. B. \& Bamshad, M. Identical mutations in NOG can cause either tarsal/carpal coalition syndrome or proximal symphalagism. Gen. Med. 3, 349-353 (2001). 5. Roth, D. A. et al. Studies in cranial suture biology. I. Increased immunoreactivity for transforming growth factor-beta $(\beta 1, \beta 2, \beta 3)$ during rat cranial suture fusion. J. Bone Miner. Res. 12, 311-321 (1997).

26. Bradley, J. P., Levine, J. P., Roth, D. A., McCarthy, J. G. \& Longaker, M. T. Studies in cranial suture biology. IV. Temporal sequence of posterior frontal cranial suture fusion in the mouse. Plast. Reconstr. Surg. 98, 1039-1045 (1996).

27. Albrecht, U., Helms, J. A. \& Lin, H. in Molecular and Cellular Methods in Developmental Toxicology (ed. Daston, G. P.) 23-48 (CRC Press, Boca Raton, 1997).

28. Paine-Saunders, S., Viviano, B. L., Economides, A. N. \& Saunders, S. Heparan sulfate proteoglycans retain noggin at the cell surface: A potential mechanism for shaping bone morphogenetic protein gradients. J. Biol. Chem. 277, 2089-2096 (2002).

Supplementary Information accompanies the paper on Nature's website ( http://www.nature.com/nature)

Acknowledgements We thank C. J. Tabin, I. Thesleff, D. M. Kingsley and N. Quarto for comments and suggestions on this manuscript, and K. D. Fong, J. A. Mathy and R. P. Nacamuli for their technical assistance. The human $f g f r 2, f g f r 2 / S 252 W$ (Apert) and $f g f r 2 / C 342 Y$ (Crouzon) retroviral constructs were provided by A. Mansukhami and C. Basilico (New York University). This work was supported by NIH grants (R.M.H. and M.T.L.) and a Lyndon Peer/PSEF Fellowship (S.M.W.)

Competing interests statement The authors declare that they have no competing financial interests.

Correspondence and requests for materials should be addressed to M.T.L. (e-mail: longaker@stanford.edu).

\section{The exocyst complex is required for targeting of Glut4 to the plasma membrane by insulin}

\section{Mayumi Inoue, Louise Chang, Joseph Hwang, Shian-Huey Chiang} \& Alan R. Saltiel

Life Sciences Institute, Departments of Internal Medicine and Physiology, University of Michigan Medical Center, Ann Arbor, Michigan 48109, USA

Insulin stimulates glucose transport by promoting exocytosis of the glucose transporter Glut4 (refs 1,2 ). The dynamic processes involved in the trafficking of Glut4-containing vesicles, and in their targeting, docking and fusion at the plasma membrane, as well as the signalling processes that govern these events, are not well understood. We recently described tyrosine-phosphorylation events restricted to subdomains of the plasma membrane that result in activation of the G protein TC10 (refs 3,4$)$. Here we show that TC10 interacts with one of the components of the exocyst complex, Exo70. Exo70 translocates to the plasma membrane in response to insulin through the activation of TC10, where it assembles a multiprotein complex that includes Sec6 and Sec8. Overexpression of an Exo70 mutant blocked insulinstimulated glucose uptake, but not the trafficking of Glut4 to the plasma membrane. However, this mutant did block the extracellular exposure of the Glut4 protein. So, the exocyst might have a crucial role in the targeting of the Glut4 vesicle to the plasma membrane, perhaps directing the vesicle to the precise site of fusion.

To search for potential effectors of TC10 that have a role in insulin-stimulated glucose transport, we screened a yeast twohybrid complementary-DNA library derived from 3T3L1 adipocytes with a constitutively active mutant form of human TC10 $\alpha^{5}$, and identified two clones encoding the full-length sequence of Exo70, a component of the exocyst complex, which has been implicated in the tethering or docking of secretory vesicles ${ }^{6,7}$. To explore the specificity of the interaction of TC10 with Exo70, we cotransfected 293T cells with myc-tagged Exo70 and haemagglutinin (HA)-tagged, constitutively active forms of mouse TC10 $\alpha$, Rho1, Rac1 or Cdc42 cDNAs ${ }^{8-11}$ (Fig. 1a). Exo70 could be co-precipitated with TC10, but not with Rho, Rac or Cdc42, indicating that its interaction with TC10 is specific. TC10 $\beta$ could also be co-precipitated with Exo70 (data not shown). To evaluate whether Exo70 is a potential effector of TC10, we incubated lysates derived from cells expressing different forms of HA-tagged TC10 with a glutathione $S$-transferase (GST)-Exo70 fusion protein, to examine binding in vitro (Fig. 1b). Exo70 bound to the constitutively active mutant $\mathrm{TC} 10 \alpha(\mathrm{Q} 67 \mathrm{~L})$, but not to the dominant-interfering TC10 $\alpha(\mathrm{T} 23 \mathrm{~N})$. Identical results were obtained with the corresponding mutants TC10ß(T25N) and TC10ß(Q69L) (data not shown). To confirm that the interaction of TC10 with Exo70 was GTP-dependent, lysates expressing TC10 were loaded with GDP or a non-hydrolysable form of GTP $(\mathrm{GTP} \gamma \mathrm{S})$ in vitro, and incubated with GST-Exo70. Exo70 bound weakly to the GDP-bound form of TC10, and strongly to the GTP-bound, activated state of the protein. These data show that Exo70 interacts with TC10 in a GTP-dependent manner.

To study the function of Exo70 in glucose transport, we sought to map its TC10 binding site through a series of deletion mutants. Exo70 has only one putative protein-protein interaction region, a coiled-coil domain in the amino terminus (Fig. 1c). We deleted amino acids 1-384 of Exo70, and also prepared constructs expressing only amino acids $1-384,1-99$ or $100-384$, and evaluated the ability of the mutants to bind constitutively active TC10 $\alpha(\mathrm{Q} 67 \mathrm{~L})$ in a co-precipitation experiment. Whereas wild-type Exo70 (Exo70WT) bound to TC10, Exo70(385-653) (Exo70-C) did not. Moreover, an Exo70 fragment containing only amino acids 1-384 (Exo70-N) bound to TC10, whereas Exo70 containing amino acids 1-99, which comprised the coiled-coil domain, bound weakly, and Exo70 containing residues 100-384 also bound weakly. These data indicate that amino acids 1-384 of Exo70 are both necessary and sufficient for binding to TC10, but that this interaction requires more than the coiled-coil domain (Fig. 1d and Supplementary Fig. 1).

We analysed the intracellular localization of Exo70 by immunohistochemistry, and evaluated its potential regulation by insulin and TC10. 3T3L1 adipocytes were transfected with myc-Exo70 plus or minus TC10 and its mutants. As shown in Fig. 2a, b, myc-Exo70 was distributed diffusely throughout the intracellular region. Coexpression of TC10 or its inactive mutant TC10(T23N) did not influence the localization of overexpressed myc-Exo70. By contrast, Exo70 was localized at the plasma membrane when co-expressed with the constitutively active mutant TC10(Q67L), indicating that TC10 activation stimulates the translocation of Exo70. We also examined whether the amino-terminal fragment of Exo70 (Exo70$\mathrm{N}$ ) translocated to the plasma membrane after expression of active TC10. Unlike the wild-type form of the protein, this fragment did 
not translocate to the membrane when co-expressed with active TC10, despite the fact that it can bind to the protein in vitro or after co-expression in $293 \mathrm{~T}$ cells (Fig. 1d). These data suggest that the carboxy-terminal sequences in Exo70 are required for the movement of the protein in adipocytes, possibly owing to an interaction with another protein.

The translocation of Exo70 to the plasma membrane by activated TC10 suggested that insulin might induce a similar redistribution of the protein. Following transfection with myc-Exo70, cells were stimulated with insulin, and the localization of the protein was examined by immunohistochemistry. Insulin stimulation resulted in the translocation of Exo70 to the plasma membrane. This event was blocked by the co-transfection of cells with the inactive mutant TC10(T23N) (Fig. 2c, d). By contrast, co-expression with the wildtype or constitutively active mutant of TC10 did not block the effect of insulin (data not shown). Interestingly, as observed above with the overexpression of active TC10, insulin failed to stimulate the translocation of the amino-terminal fragment of Exo70 to the plasma membrane.

The observation that insulin can recruit Exo70 to the plasma membrane by way of TC10 suggests that insulin might promote the assembly of the exocyst complex through an interaction between TC10 and Exo70. The exocyst complex is thought to assemble before the fusion of secretory vesicles with the plasma membrane ${ }^{12-14}$. This complex includes the proteins Sec6 and Sec8, which have been shown to regulate the late stage of exocytosis in MDCK cells ${ }^{15,16}$. Unfortunately, antibodies to Sec6 and Sec8 could not be used for a

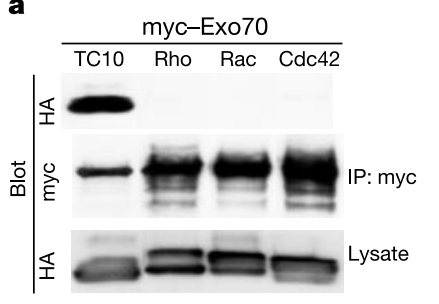

d

b ${ }_{\text {HA-TC10 } \alpha} \frac{\text { GST-Exo70 }}{\text { Q67LT23N WT }}$ In vitro loading $\frac{\mathrm{WT}}{+\mathrm{GDP}+\mathrm{GTP} \gamma \mathrm{S}}$
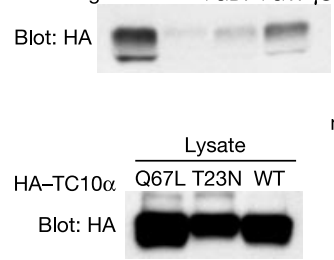

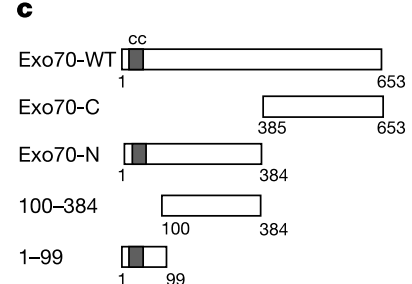
myc-Exo70 WT 1-384 385-653 Control
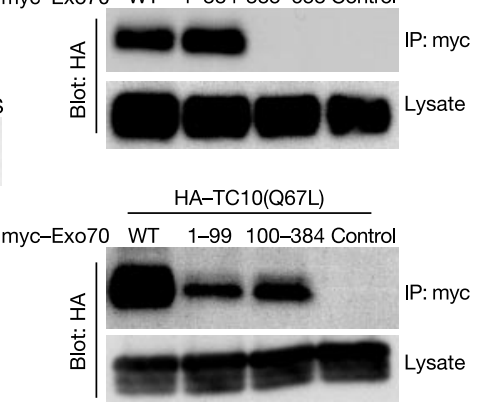

Figure 1 Ex070 interacts with activated TC10. a, Ex070 specifically interacts with TC10. 293T cells were co-transfected with myc-Exo70 and HA-tagged, constitutively active forms of $\mathrm{TC10} \alpha(\mathrm{Q} 67 \mathrm{~L}), \operatorname{RhoA}(\mathrm{G} 14 \mathrm{~V})$, Rac1(G12V) and Cdc42(Q61L). Lysates were immunoprecipitated (IP) with an anti-myc antibody, followed by blotting with anti-HA antibody. b, Ex070 interacts with TC10 in a GTP-dependent manner. 293T cells were transfected with HA-tagged wild-type TC10 $\alpha$, its constitutively active Q67L mutant, or its inactive T23N mutant. Cell lysates were preincubated with either $100 \mu \mathrm{M}$ GTP $\gamma$ S or $1 \mathrm{mM}$ GDP, and then incubated with glutathione-Sepharose-bound GST-Ex070. Precipitates were analysed by immunoblot analysis with anti-HA antibody. Identical results were obtained with TC10 (data not shown). c, Schematic of Ex070 and its deletion mutants. cc, coiled-coil. d, Interaction of TC10 with Exo70 mutants. 293T cells were co-transfected with $\mathrm{HA}-\mathrm{TC10} \alpha(\mathrm{Q} 67 \mathrm{~L})$ and myc-tagged wild-type Ex070 or mutants containing amino acids 1-384 (Exo70-N), 385-653 (Exo70-C), 1-99, 100-384 or empty vector. Lysates were immunoprecipitated with anti-myc antibody, and immunopellets were analysed by blotting with anti-HA antibody. immunohistochemistry. So, to evaluate the localization of these endogenous proteins, we fractionated cell lysates derived from 3T3L1 adipocytes stimulated with or without insulin. Sec6 and Sec8 were found in cytosolic and low-density microsomal (LDM) fractions, with a small amount found in the plasma-membrane fraction. Treatment of cells with insulin reduced the appearance of the two proteins in the cytoplasm and low-density microsomes, with a corresponding increase in the plasma-membrane fraction (Fig. 3a).

We also evaluated whether Exo70 formed a complex with Sec6 and Sec8. As shown in Fig. 3b, endogenous Sec6 bound to Sec8, as assayed by co-precipitation. This interaction was insulin-indepen-

a myc-Exo70-WT myc-Exo70-N
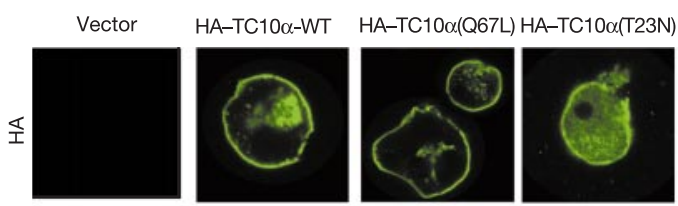
$\mathrm{HA}-\mathrm{TC} 10 \alpha(\mathrm{Q} 67 \mathrm{~L})$
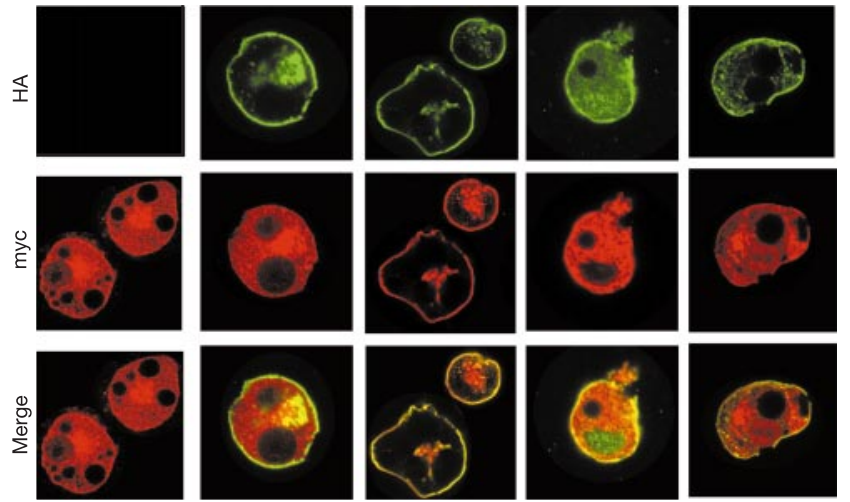

b
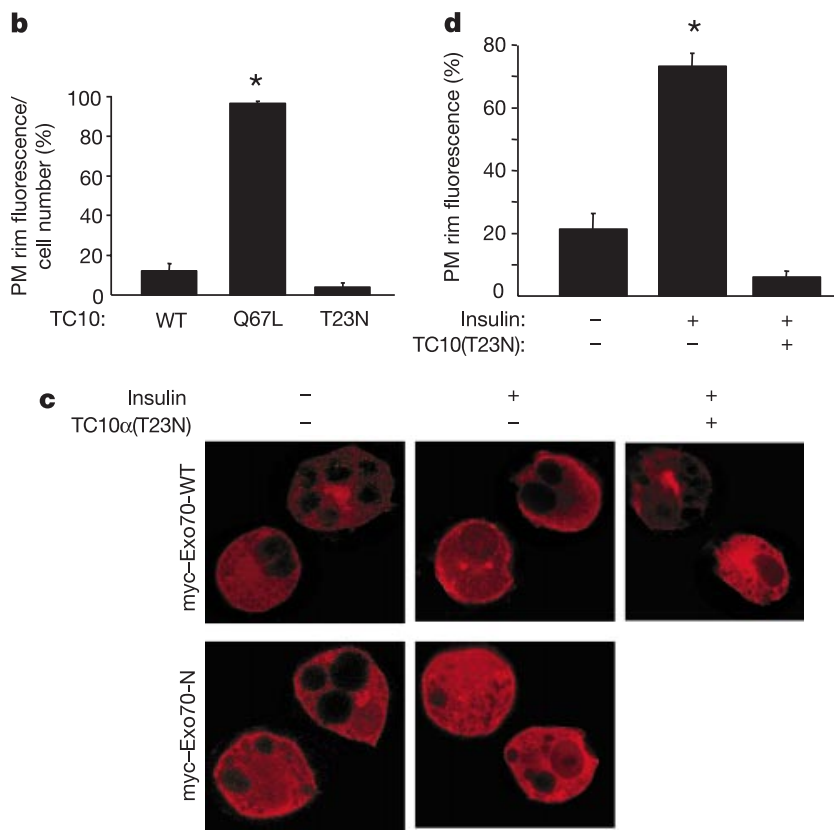

Figure 2 Ex070 is translocated to the plasma membrane by insulin. a, Overexpression of constitutively active $\mathrm{TC10}(\mathrm{Q} 75 \mathrm{~L})$ recruits Exo70 to the plasma membrane (PM). 3T3L1 adipocytes were co-transfected with myc-Exo70-WT or myc-Exo70-N with vector only, HA-tagged wild-type TC10, or its Q75L or T31N mutants. Cells were fixed and stained with anti-myc polyclonal antibody to detect myc-Exo70, and with anti-HA monoclonal antibody to detect HA-TC10, followed by Alexa ${ }^{594}$ goat anti-rabbit lgG and Alexa ${ }^{488}$ goat anti-mouse lgG. Fluorescence was visualized by confocal laser scanning microscopy. Images represent four independent determinations. b, d, Graphical representation of data from three independent experiments. *Significant difference, $P<0.0001$. c, Ex070 translocates to the plasma membrane in response to insulin. 3T3L1 adipocytes were electroporated with myc-Ex070-WT or myc-Exo70-N with or without HA-TC10(T31N), serum-starved, and treated with or without insulin for $5 \mathrm{~min}$. Cells were fixed and stained with anti-myc antibody followed by Alexa ${ }^{594}$ goat anti-mouse IgG. 
dent. Moreover, both wild-type Exo70 and its amino-terminal fragment bound to endogenous Sec8 (Fig. 3c, d). Interestingly, whereas the amino-terminal region of Exo70 bound to Sec8, a fragment of the protein containing only amino acids 385-653 did not (data not shown). So, Exo70 appears to exist in a preformed complex with Sec6 and Sec8, through an interaction in the aminoterminal region of Exo70. This multiprotein complex is then translocated to the plasma membrane in response to insulin.

As the amino-terminal fragment of Exo70 fails to translocate to the plasma membrane in response to insulin or active TC10, this mutant form of the protein might also block the assembly of the exocyst complex at the cell surface. Cells were transfected with the myc-tagged wild-type or amino-terminal fragment of Exo70 (Fig. 3e), treated with insulin, and fractionated as detailed above. As determined by immunohistochemical analysis (Fig. 2), insulin stimulated the translocation of wild-type Exo70 to the plasma membrane. However, the amino-terminal fragment remained mainly in the cytoplasm, and the localization of this protein was not influenced by insulin. Sec8 also translocated to the membrane in response to insulin in cells that expressed wild-type Exo70.
However, expression of the mutant form of the protein blocked the translocation of endogenous Sec8. These data indicate that the amino-terminal fragment of Exo70 can act as a dominant-negative mutant, presumably blocking the insulin-stimulated assembly of the exocyst complex at the plasma membrane.

The exocyst complex is thought to have an important role in targeted exocytosis. To evaluate whether the interaction between TC10 and Exo70 is involved in Glut4 vesicle trafficking, we overexpressed Exo70 and its mutants in 3T3L1 adipocytes by electroporation, and assayed 2-deoxyglucose uptake ${ }^{17}$ (Fig. 4a). In cells transfected with an empty vector, insulin produced a fivefold increase in 2-deoxyglucose uptake. Overexpression of wild-type Exo70 led to a $48 \%$ increase in insulin-stimulated glucose uptake over that observed in cells transfected with an empty vector, although basal activity was not significantly changed. By contrast, the amino-terminal fragment of Exo70, which blocks the assembly of the exocyst complex, produced a $40 \%$ inhibition of insulinstimulated transport compared with control, although basal activity was again unaffected. In these experiments, less than $50 \%$ of the cells were transfected by the electroporation method, indicating that a
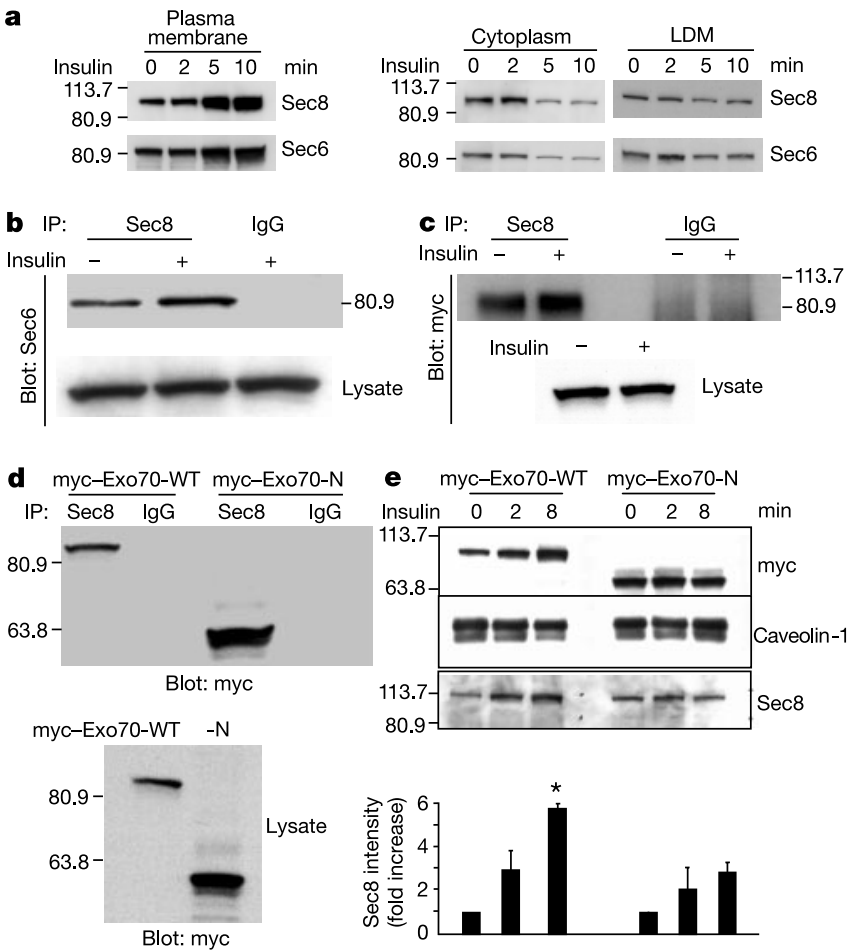

80.9

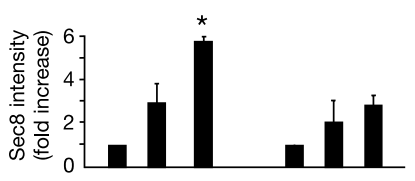

Figure 3 Components of the exocyst complex translocate to the plasma membrane in response to insulin. a, Sec6 or Sec8 translocate to the plasma membrane in response to insulin stimulation. After insulin stimulation, cells were placed on ice and washed three times with ice-cold PBS. Membrane fractionation was carried out using 3T3L1 adipocytes as reported ${ }^{29}$, and endogenous Sec6 and Sec8 were detected by monoclonal antibodies. b, Sec8 interacts with Sec6. The cell lysates from 3T3L1 adipocytes were incubated with a Sec8 monoclonal antibody and blotted with a Sec6 antibody. c, Sec8 and Exo70 interact. 3T3L1 adipocytes were transfected with myc-Ex070. Cells were stimulated with $100 \mathrm{nM}$ insulin for 10 min. Cell lysates were incubated with a Sec8 monoclonal antibody and blotted with an anti-myc polyclonal antibody. d, Sec8 interacts with Exo70-WT and Exo70-N. Lysates were prepared as described in c from 3T3L1 adipocytes transfected with myc-Ex070-WT or myc-Exo70-N. e, Exo70-N blocks membrane translocation of Sec8. Membrane fractionation was carried out using 3T3L1 adipocytes transfected with myc-Ex070-WT and myc-Exo70-N after insulin stimulation. Endogenous Sec8 and caveolin- 1 were detected by western blotting using the plasma-membrane fraction. The means (and standard deviations) of three independent determinations are shown in graphical form. *Significant difference, $P<0.005$.
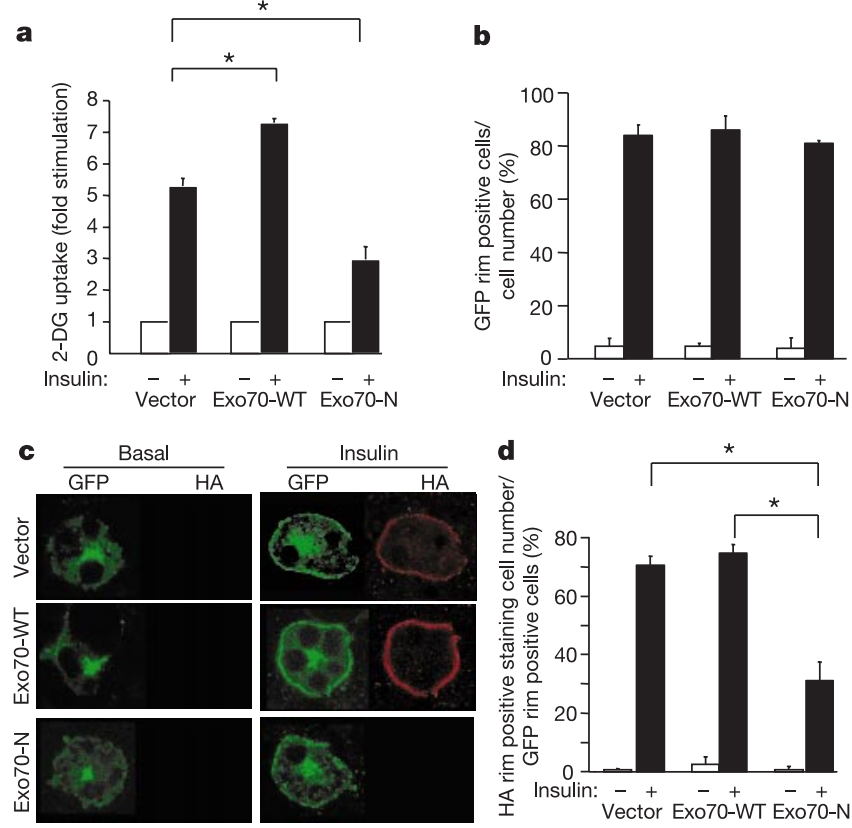

Figure 4 Exo70 plays a crucial role in Glut4 vesicle targeting. a, Differentiated 3T3L1 adipocytes were transfected with Ex070-WT or Ex070-N. The cells were left untreated or were stimulated with $100 \mathrm{nM}$ insulin for $30 \mathrm{~min}$. The rate of $\left[{ }^{14} \mathrm{C}\right] 2$-deoxyglucose (2-DG) uptake was determined. Cells were transfected with an efficiency of approximately $50 \%$. Results are expressed as the mean \pm s.d. of five separate experiments. *Significant difference, $P<0.01$. b, Number of Glut4-eGFP transfected cells with visually detectable plasma-membrane rim fluorescence. The numbers of Glut4-eGFP-transfected cells displaying visually detectable eGFP rim fluorescence are plotted. c, Exo70-N does not affect HA-Glut4-eGFP translocation, but blocks its extracellular exposure. 3T3L1 adipocytes were co-electroporated with HA-Glut4-eGFP plus vector alone, myc-tagged Exo70-WT or Exo70-N. Cells were serum-starved, treated with or without insulin for $30 \mathrm{~min}$, fixed, and stained with an anti-HA monoclonal antibody followed by Alexa ${ }^{594}$ goat anti-mouse IgG to detect cells that expressed HA-Glut4 at the surface. Fluorescence was visualized by confocal laser scanning microscopy. Images represent three independent determinations. d, Percentage of the HA rim positive staining cells among the HA-Glut4-GFP-transfected cells was quantified. Fifty cells that showed translocation of eGFP were scored for visually detectable HA rim fluorescence before and after treatment with insulin for $30 \mathrm{~min}$. These data were obtained by blind counting of more than 50 cells from three independent experiments. *Significant difference, $P<0.0001$. 
most of the insulin-stimulated glucose transport was blocked by overexpression of the Exo70-N mutant.

As mentioned above, insulin-stimulated glucose transport results from the translocation of the Glut4 protein to the cell surface. So, we next evaluated the effect of Exo70 on the translocation of a Glut4enhanced green fluorescent protein (eGFP) fusion protein in 3T3L1 adipocytes. Cells were co-transfected with Glut4-eGFP and with wild-type or mutant forms of Exo70. Insulin stimulated the translocation of Glut4-eGFP in $90 \%$ of the cells transfected with vector alone, as detected by increased rim fluorescence. Co-expression with wild-type Exo70 had no impact on basal or insulin-stimulated translocation. Interestingly, co-expression with the amino-terminal fragment of Exo70 was also without effect on Glut4-eGFP translocation, despite the clear inhibition of glucose transport observed by overexpression of this mutant (Fig. 4b).

The inhibition of insulin-stimulated glucose uptake in the face of no effect on Glut 4 translocation by the Exo70 mutant indicated that the protein might influence the targeting or docking of Glut4 to the plasma membrane, rather than the translocation process. To explore this possibility in more detail, we co-expressed the Exo70 mutants with a Glut4-GFP construct that was HA-tagged in its first extracellular loop ${ }^{18}$ (Fig. 4c). This double-tagged form of Glut4 can be detected by the fluorescence of GFP, and simultaneously by immunodetection of the HA tag at the cell surface in nonpermeabilized cells, thus representing a method to evaluate both the translocation and extracellular exposure of Glut4 (ref. 18). In the absence of insulin, there was no cell-surface staining of HA-Glut4GFP with anti-HA, whereas insulin treatment produced a strong signal. Co-transfection of cells with wild-type Exo70 significantly increased the cell-surface staining of HA-Glut4-GFP, as detected by anti-HA antibodies, consistent with the increase in glucose transport produced by overexpression of the protein. By contrast, cotransfection of cells with the amino-terminal fragment of Exo70 completely blocked the appearance of HA-Glut4-GFP at the cell surface (Fig. 4c, d), indicating that Exo70 might have a crucial role in the membrane targeting of Glut4. An inhibitory mutant of TC10 also blocked the cell-surface exposure of exofacial tagged Glut4 (Supplementary Fig. 2).

The translocation of the insulin-responsive glucose transporter Glut4 from intracellular membranes to the cell surface is likely to involve numerous steps, typical of vesicular transport in other systems. These may include mechanisms for the tethering of the vesicle to target membranes, where it can come into contact with the docking and fusion machinery. For many exocytotic processes, this step is mediated by the exocyst, an evolutionarily conserved octameric complex that targets vesicles to specific sites of the plasma membrane ${ }^{19}$. Data presented here suggest that the exocyst complex assembles at the plasma membrane of fat cells in response to insulin, through the association of Exo70 with activated TC10, and has a role in the tethering of the Glut4 vesicle, where it can come into proximity with the SNARE (SNAP receptor) complexes involved in docking and fusion ${ }^{20,21}$. The Rab proteins, which are mammalian homologues of the yeast exocyst component Sec4, might mediate this tethering step. Rab4 has been found in Glut4 vesicles, and translocates to the plasma membrane in response to insulin ${ }^{22-25}$. Moreover, we have also shown that TC10 can interact with the effector protein CIP4, which is required for Glut4 translocation ${ }^{26}$. So, TC10 might engage multiple effectors that influence both Glut4 translocation and tethering of the vesicle at the plasma membrane. This possibility is under investigation.

\section{Methods}

\section{Yeast two-hybrid screen}

The cDNA encoding full-length human TC10(Q75L) was amplified by polymerase chain reaction (PCR) using the plasmid pKH3-TC10(Q75L) (a gift from I. Macara, University of Virginia, Charlottesville) as a template, and a yeast two-hybrid cDNA library derived from 3T3L1 adipocyte messenger RNA was screened as described previously ${ }^{26}$.

\section{Expression constructs}

To generate a mammalian expression vector, full-length Exo70 cDNA was subcloned into the pCS2 vector. To construct deletion mutants, Exo70 cDNA was digested with $\mathrm{NcoI}$ and religated, generating Exo70-N or Exo70-C mutants. For 1-99 or 100-384 mutants, Exo70 was PCR-amplified from pCS2-Exo70 using oligonucleotides containing EcoRI/XhoI restriction sites, and subcloned into pCS2 at EcoRI/XhoI.

\section{In vitro GST pull-down assay}

GST or GST-Exo70 fusion proteins were expressed in the BL21(DE3) pLysS Escherichia coli strain and purified. Cell lysates were prepared for immunoprecipitation as described below, and incubated with either GST alone or GST-Exo70 bound to glutathioneSepharose beads (Amersham Pharmacia) for $2 \mathrm{~h}$ at $4{ }^{\circ} \mathrm{C}$. For GTP-binding experiments, cell lysates were loaded in vitro with either $1 \mathrm{mM}$ GDP or $100 \mu \mathrm{M} \mathrm{GTP} \gamma \mathrm{S}$ for $30 \mathrm{~min}$ at $37^{\circ} \mathrm{C}$ before incubating with GST fusion protein $\mathrm{s}^{27}$. The beads were washed five times with lysis buffer, resuspended in sample buffer, subjected to SDS-polyacrylamide gel electrophoresis (SDS-PAGE), and analysed by immunoblotting with anti-myc monoclonal antibody or polyclonal antibody (Santa Cruz Biotechnology) or anti-HA monoclonal antibody (Santa Cruz Biotechnology).

\section{Immunoprecipitation and immunoblotting}

Cell lysates were prepared as described using 1\% Triton X-100 (ref. 4). Lysates were then incubated with the indicated antibodies for $2 \mathrm{~h}$ at $4^{\circ} \mathrm{C}$. The immune complexes were precipitated with protein $\mathrm{A}$ or $\mathrm{G}$ agarose (Amersham Pharmacia) for $1 \mathrm{~h}$ at $4{ }^{\circ} \mathrm{C}$, washed extensively with lysis buffer, resolved in 10-20\% gradient SDS-PAGE, and analysed by immunoblotting. All immunoblots were developed by enhanced chemiluminesence (Amersham Pharmacia). Anti-Sec6 (9H5) and Sec8 (2E9) monoclonal antibodies were obtained from Calbiochem, and anti-caveolin-1 polyclonal antibody was obtained from Transduction Laboratories.

\section{Immunofluorescence microscopy}

For immunofluorescence studies, electroporated cells were processed as described ${ }^{17}$. To detect myc-Exo70, cells were stained with anti-myc monoclonal antibody or polyclonal antibody (Santa Cruz Biotechnology). To detect HA-TC10, cells were stained with antiHA monoclonal antibody (Santa Cruz Biotechnology). After incubation with primary antibodies, cells were incubated with Alexa ${ }^{488}$ or Alexa ${ }^{594}$ goat anti-mouse or anti-rabbit immunoglobulin $(\operatorname{IgG})$, obtained from Molecular Probes. Images were captured by using an Olympus FV300 confocal laser scanning microscope.

\section{Assays of Glut4-eGFP translocation and HA-Glut4-GFP}

Glut4-eGFP translocation assays were performed as described ${ }^{28}$. The HA-Glut4-GFP construct was a gift from S. W. Cushman (National Institutes of Health, Maryland). The method was followed as described previously ${ }^{18}$.

Received 25 November 2002; accepted 4 March 2003; doi:10.1038/nature01533.

1. Saltiel, A. R. \& Kahn, C. R. Insulin signalling and the regulation of glucose and lipid metabolism. Nature 414, 799-806 (2001).

2. Bryant, N. J., Govers, R. \& James, D. E. Regulated transport of the glucose transporter GLUT4. Nature Rev. Mol. Cell Biol. 3, 267-277 (2002).

3. Chiang, S. H. et al. Insulin-stimulated GLUT4 translocation requires the CAP-dependent activation of TC10. Nature 410, 944-948 (2001).

4. Liu, J., Kimura, A., Baumann, C. A. \& Saltiel, A. R. APS facilitates c-Cbl tyrosine phosphorylation and GLUT4 translocation in response to insulin in 3T3-L1 adipocytes. Mol. Cell. Biol. 22, 3599-3609 (2002).

5. Neudauer, C. L., Joberty, G., Tatsis, N. \& Macara, I. G. Distinct cellular effects and interactions of the Rho-family GTPase TC10. Curr. Biol. 8, 1151-1160 (1998).

6. TerBush, D. R., Maurice, T., Roth, D. \& Novick, P. The exocyst is a multiprotein complex required for exocytosis in Saccharomyces cerevisiae. EMBO J. 15, 6483-6494 (1996).

7. Kee, Y. et al. Subunit structure of the mammalian exocyst complex. Proc. Natl Acad. Sci. USA 94, 14438-14443 (1997).

8. Symons, M. Rho family GTPases: the cytoskeleton and beyond. Trends Biochem. Sci. 21, 178-181 (1996).

9. Tapon, N. \& Hall, A. Rho, Rac, and Cdc42 GTPases regulate the organization of the actin cytoskeleton. Curr. Opin. Cell Biol. 9, 86-92 (1997).

10. Van Aelst, L. \& D'Souza-Schorey, C. Rho GTPases and signaling networks. Genes Dev. 11, 2295-2322 (1997)

11. Chiang, S. H., Hou, J. C., Hwang, J., Pessin, J. E. \& Saltiel, A. R. Cloning and functional characterization of related TC10 isoforms, a subfamily of Rho proteins involved in insulin-stimulated glucose transport. J. Biol. Chem. 277, 13067-13073 (2002).

12. Hsu, S. C., Hazuka, C. D., Foletti, D. L. \& Scheller, R. H. Targeting vesicles to specific sites on the plasma membrane: the role of the sec6/8 complex. Trends Cell. Biol. 9, 150-153 (1999).

13. Guo, W., Sacher, M., Barrowman, J., Ferro-Novick, S. \& Novick, P. Protein complexes in transport vesicle targeting. Trends Cell Biol. 10, 251-255 (2000).

14. Lipschutz, J. H. \& Mostov, K. E. Exocytosis: the many masters of the exocyst. Curr. Biol. 12, R212-R214 (2002).

15. Ting, A. E. et al. rSec6 and $\mathrm{rSec} 8$, mammalian homologs of yeast proteins essential for secretion. Proc. Natl Acad. Sci. USA 92, 9613-9617 (1995)

16. Yeaman, C., Grindstaff, K. K., Wright, J. R. \& Nelson, W. J. Sec6/8 complexes on trans-Golgi network and plasma membrane regulate late stages of exocytosis in mammalian cells. J. Cell Biol. 155, 593-604 (2001)

17. Kimura, A., Baumann, C. A., Chiang, S. H. \& Saltiel, A. R. The sorbin homology domain: a motif for the targeting of proteins to lipid rafts. Proc. Natl Acad. Sci. USA 98, 9098-9103 (2001).

18. Dawson, K., Aviles-Hernandez, A., Cushman, S. W. \& Malide, D. Insulin-regulated trafficking of duallabelled glucose transporter 4 in primary rat adipose cells. Biochem. Biophys. Res. Commun. 287, 445-454 (2001). 
19. Novick, P. \& Guo, W. Ras family therapy: Rab, Rho and Ral talk to the exocyst. Trends Cell Biol. 12 $247-249$ (2002).

20. Cheatham, B. GLUT4 and company: SNAREing roles in insulin-regulated glucose uptake. Trends Endocrinol. Metab. 11, 356-361 (2000).

21. Chamberlain, L. H. \& Gould, G. W. The v-and t-SNARE proteins that mediate Glut4 vesicle fusion are localised in detergent-insoluble lipid rafts present on distinct intracellular membranes. J. Biol. Chem. 277, 49750-49754 (2002)

22. Sherman, L. A., Hirshman, M. F., Cormont, M., Le Marchand-Brustel, Y. \& Goodyear, L. J. Differentia effects of insulin and exercise on Rab4 distribution in rat skeletal muscle. Endocrinology 137, 266-273 (1996).

23. Cormont, M. et al. Potential role of Rab4 in the regulation of subcellular localization of Glut4 in adipocytes. Mol. Cell. Biol. 16, 6879-6886 (1996).

24. Shisheva, A. \& Czech, M. P. Association of cytosolic Rab4 with GDI isoforms in insulin-sensitive 3T3L1 adipocytes. Biochemistry 36, 6564-6570 (1997)

25. Millar, C. A., Shewan, A., Hickson, G. R., James, D. E. \& Gould, G. W. Differential regulation of secretory compartments containing the insulin-responsive glucose transporter 4 in 3T3-L1 adipocytes. Mol. Biol. Cell 10, 3675-3688 (1999).

26. Chang, L., Adams, R. D. \& Saltiel, A. R. The TC10-interacting protein CIP4/2 is required for insulinstimulated Glut4 translocation in 3T3L1 adipocytes. Proc. Natl Acad. Sci. USA 99, 12835-12840 (2002).

27. Lin, D. et al. A mammalian PAR-3-PAR-6 complex implicated in Cdc42/Racl and aPKC signalling and cell polarity. Nature Cell Biol. 2, 540-547 (2000)

28. Thurmond, D. C. et al. Regulation of insulin-stimulated GLUT4 translocation by Munc18c in 3T3L1 adipocytes. J. Biol. Chem. 273, 33876-33883 (1998).

29. Hwang, J. B. \& Frost, S. C. Effect of alternative glycosylation on insulin receptor processing. J. Biol. Chem. 274, 22813-22820 (1999).

Supplementary Information accompanies the paper on Nature's website ( http://www.nature.com/nature).

Acknowledgements This work was supported by grants from the National Institutes of Health We thank T-H. Chun for helpful discussions.

Competing interests statement The authors declare that they have no competing financial interests.

Correspondence and requests for materials should be addressed to A.R.S. (e-mail: saltiel@umich.edu).

\section{Noise in eukaryotic gene expression}

\author{
William J. Blake*, Mads Kærn*, Charles R. Cantor \& J. J. Collins \\ Center for BioDynamics, Center for Advanced Biotechnology, Bioinformatics \\ Program, and Department of Biomedical Engineering, Boston University, \\ 44 Cummington Street, Boston, Massachusetts 02215, USA \\ * These authors contributed equally to this work
}

Transcription in eukaryotic cells has been described as quantal ${ }^{1}$, with pulses of messenger RNA produced in a probabilistic manner $^{2,3}$. This description reflects the inherently stochastic nature $^{4-9}$ of gene expression, known to be a major factor in the heterogeneous response of individual cells within a clonal population to an inducing stimulus ${ }^{10-16}$. Here we show in Saccharomyces cerevisiae that stochasticity (noise) arising from transcription contributes significantly to the level of heterogeneity within a eukaryotic clonal population, in contrast to observations in prokaryotes ${ }^{15}$, and that such noise can be modulated at the translational level. We use a stochastic model of transcription initiation specific to eukaryotes to show that pulsatile mRNA production, through reinitiation, is crucial for the dependence of noise on transcriptional efficiency, highlighting a key difference between eukaryotic and prokaryotic sources of noise. Furthermore, we explore the propagation of noise in a gene cascade network and demonstrate experimentally that increased noise in the transcription of a regulatory protein leads to increased cell-cell variability in the target gene output, resulting in prolonged bistable expression states. This result has implications for the role of noise in phenotypic variation and cellular differentiation.
To explore the effects of transcriptional variation and control on the level of noise in eukaryotic gene expression, we used both native and artificial modes of transcriptional regulation in the yeast GAL1 promoter (Fig. 1a). In its natural context, the GAL1 promoter is activated in response to galactose (in the absence of preferentially metabolized glucose) through an upstream activation sequence $\left(\mathrm{UAS}_{\mathrm{G}}\right.$ ) composed of multiple binding sites for the transcriptional activator Gal4p. Like many eukaryotic activators ${ }^{17}$, Gal4p acts by recruiting protein complexes involved in chromatin remodelling and the ordered assembly of a transcription preinitiation complex $^{18,19}$. Because Gal4p is a galactose-dependent transcriptional activator, activation-based expression from the GAL1 promoter is effectively modulated with galactose. As a second mode of transcriptional control, distinct from the native complexity of the yeast galactose-utilization pathway, we constructed an artificial, Tet-responsive GAL1 promoter $\left(\mathrm{P}_{\mathrm{GAL1}^{*}}\right)$ by inserting tandem tet operators $\left(2 \times t_{t e t O}\right)$ downstream of the GAL1 TATA box. In contrast to Gal4p-mediated activation, bound Tet repressor (TetR) might act by sterically hindering the assembly of the transcriptional machinery, effectively repressing expression from $\mathrm{P}_{\mathrm{GAL} 1^{*}}$. TetRmediated repression can be relieved by the addition of the chemical inducer anhydrotetracycline (ATc), which binds directly to TetR. Constitutive expression of TetR therefore allows rheostat-like control of $\mathrm{P}_{\mathrm{GAL}^{*}}$ transcriptional efficiency through the use of varying levels of ATc. The gene encoding the yeast-enhanced green fluorescent protein $(y E G F P)$ was expressed from $\mathrm{P}_{\mathrm{GAL} 1^{*}}$ as a quantifiable reporter, and fluorescence histograms were obtained from flow cytometric measurement of similarly sized cells containing chromosomally integrated, single genetic copies of each construct.

Transcription from $\mathrm{P}_{\mathrm{GAL} 1^{*}}$ is modulated over a broad dynamic range by both native and artificial modes of regulation (Fig. 1b), allowing a direct comparison between galactose- and ATc-mediated

$\mathbf{a}$
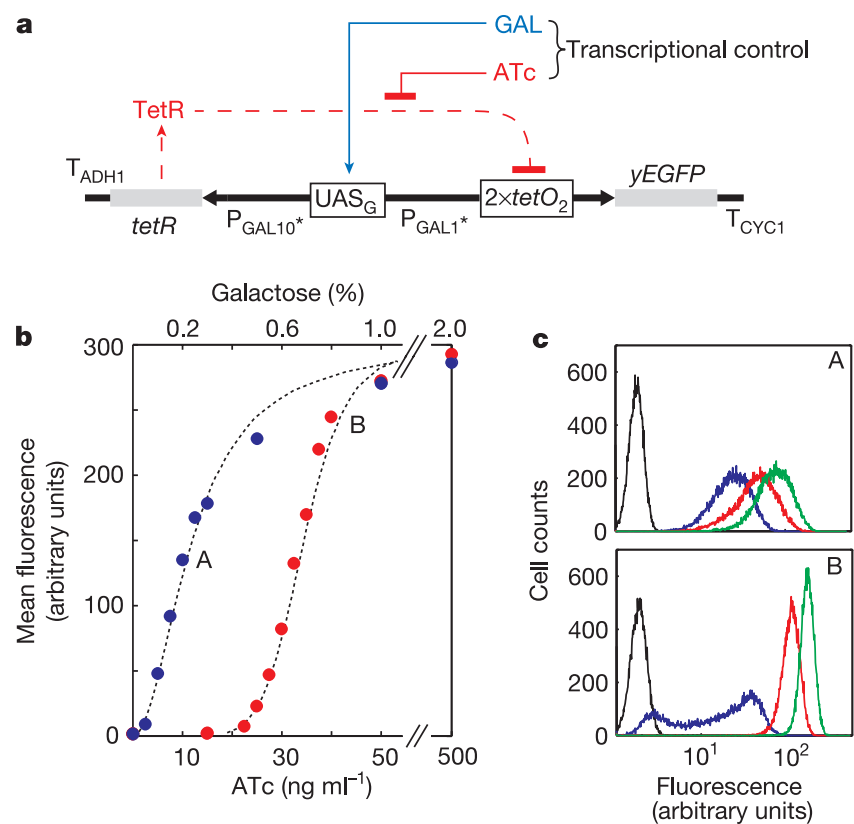

Figure 1 Transcriptional control of $P_{G A L 1^{*}}$ a, TetR, expressed from $P_{G A L 10^{*}}$, represses expression of $y E G F P$. Anhydrotetracycline (ATC) and galactose (GAL) are required to induce yEGFP expression. Transcription terminators $\left(\mathrm{T}_{\mathrm{ADH1}}, \mathrm{T}_{\mathrm{CYC1}}\right)$ are indicated. b. Dose-response curve of $P_{G A L}$ * expressing yEGFP to ATC at full galactose induction (2\%; red points), and to galactose at full ATc induction (500 $\mathrm{ng} \mathrm{ml}^{-1}$; blue points). Broken lines were obtained from stochastic simulations (Box 1). c, Transient response of cells marked $A$ and $B$ in $\mathbf{b}$, induced with $0.2 \%$ galactose and $40 \mathrm{ng} \mathrm{ml}^{-1} \mathrm{ATc}$, respectively. Histograms correspond to preinduction (black), and $150 \mathrm{~min}$ (blue), $290 \mathrm{~min}$ (red) and 440 min (green) after induction. 